\title{
CODE STEMI Improves Clinical Outcomes and Cost Efficiency in ST Elevation Myocardial Infarction Patients : A Retrospective Cohort Study
}

Eka Ginanjar ( $\sim$ ekginanjar@gmail.com )

Rumah Sakit Dr Cipto Mangunkusumo https://orcid.org/0000-0002-5671-9167

Amal Chalik Sjaaf

Faculty of Public Health University of Indonesia

Idrus Alwi

Rumah Sakit Dr Cipto Mangunkusumo

Wahyu Sulistyadi

Faculty of Public Health University of Indonesia

\section{Ede Suryadarmawan}

Faculty of public Health University of Indonesia

\section{Adik Wibowo}

Faculty of Public Health University of Indonesia

\section{Lies Dina Liastuti}

Rumah Sakit Dr Cipto Mangunkusumo

\section{Research article}

Keywords: myocardial infarction, CODE STEMI, MACE,mortality, cost-efficiency, percutaneous coronary intervention

Posted Date: May 15th, 2020

DOI: https://doi.org/10.21203/rs.3.rs-25997/v1

License: (1) (i) This work is licensed under a Creative Commons Attribution 4.0 International License. Read Full License 


\section{Abstract}

Background One of the highest causes of cardiac mortality is ST Elevation Myocardial Infarction (STEMI). Delay in the management of STEMI patients resulted high mortality, morbidity, and economic burden. CODE STEMI is a protocol to reduce door to balloon time and improve patient's quality care and clinical outcome. This study aims to determine the effect of the implementation of CODE STEMI on clinical outcome and cost efficiency of a quality care of STEMI patients at Dr. Cipto Mangunkusumo General Hospital.

Method This is a retrospective cohort study that reviewed 207 medical records of STEMI patients who underwent primary percutaneous coronary intervention (PPCl) in 2015-2018. The patients were divided into two groups. The first group was treated prior to establishing the CODE STEMI program. The other group was treated according to the program, which was implemented in January 2017. The primary outcome was to measure in-hospital mortality and morbidity and total hospital cost between both groups. Secondary end-points included length of stay (LOS) and Cost Efficient Analysis. Data analysis was done using Mann Whitney and Chi square test.

Results There were 72 and 135 patients in Pre-CODE STEMI and CODE STEMI groups respectively. In CODE STEMI group, median D2BT was significantly reduced by $130 \mathrm{~min}(\mathrm{P}<0.001)$, median LOS was reduced by 1 day $(P=0.009)$ and there was cost reduction by approximately USD 1,000 . There were declining trend of in-hospital mortality rates (8.3\% vs $4.4 \%)$ and MACE at 30 days (48.61\% vs $37.78 \%$ ).

Conclusion Implementation of CODE STEMI can improve clinical outcome, reduce length of stay and cost efficiency of STEMI patients in general hospitals.

\section{Introduction}

Coronary heart disease (CHD) is the leading cause of death worldwide ${ }^{1}$ and is one of the leading causes of disease burden in developing countries. ${ }^{2}$ The WHO (World Health Organization) report in 2017 estimated around 17.8 million death are caused by cardiovascular disease each year. ${ }^{3}$ Coronary heart disease (CHD) contributed to half cases of death from cardiovascular diseases. CHD has various clinical manifestations ranging from asymptomatic to acute conditions, namely acute coronary syndrome (ACS) including ST Elevation Myocardial Infarction (STEMI), Non ST Elevation Myocardial Infarction (NSTEMI) and unstable angina pectoris (UAP). Based on the Euro Heart Survey in 2004, mortality rates related to STEMI in Europe reached $6-14 \%{ }^{4}$

The majority of cases in ST Elevation Myocardial Infarction (STEMI) occurs mainly because of the occlusion of the main coronary arteries. Management of STEMI must be done as soon as possible to prevent further damage to the heart muscles and lower the risk of complication and mortality in patients with STEMI. Reperfusion/revascularization therapy plays a pivotal role in the management of ACS to treat the etiology and improve the clinical condition of patients. ${ }^{5}$ Timely reperfusion with percutaneous Loading [MathJax]/jax/output/CommonHTML/fonts/TeX/fontdata.js 
coronary intervention ( $\mathrm{PCl}$ ) within 12 hours of symptom onset is currently the treatment of choice in STsegment-elevation. ${ }^{6}$ Therefore, delay in performing PCI for STEMI patients is the cause of high mortality and the incidence of MACE (Major Adverse Cardiac Event).

Cardiovascular disease exerts a significant economic toll, accounting for one third of a projected $\$ 47$ trillion in economic losses to noncommunicable diseases (NCDs) for the next 20 years. ${ }^{7}$ Aside from associated with substantial healthcare burden worldwide, the costs of treating ACS also represent major burden for healthcare system globally. ${ }^{8}$ Evidence from the US, comparing inpatient resource use for ACS in patients who died in hospital with that for a surviving ACS cohort, indicated that inpatient mortality for ACS is associated with a $47 \%$ greater duration of hospital stay along with an incremental cost of around US\$43,000. ${ }^{9}$ This situation might have significant impact on the sustainability of health financing systems, particularly in developing countries in Asia.

In general hospitals, the problem that can be found frequently is the lack of health workers' awareness of the importance of immediate treatment for STEMI patients. This problem starts from the Emergency Room (ED). ED crowding has become an issue of great concern in general hospitals, especially since the amount of health workers and facilities are not proportional to the number of patients that come with different complaints. Moreover, many patients do not want to cooperate easily, adding to the strenuous job of health care workers, which resulted in chaotic order in the patients' management, including STEMI patients. There were five potential problems in managing STEMI in daily practices, such as patient delay, diagnosis and treatment decision delay, transportation delay, and lack of collaboration between hospital management and doctors. ${ }^{10}$ All of these resulted in the delay of door-to-balloon time (D2BT), which in turn increased the risk of MACE and mortality, and prolong the hospital stay. All of this will contribute to higher treatment cost.

An establishment of CODE STEMI, an ED physician-activated STEMI notification system, is expected to help solve this issue. CODE STEMI is a protocol where a standard integrated clinical pathway is made especially for STEMI patients, including a policy that monitors every incident in handling STEMI patients. The aim is to shorten the D2BT, the time needed for patient to receive immediate reperfusion therapy (primary percutaneous coronary intervention) from initial arrival at the hospital. Previous study has proven that reducing door-to-balloon time in STEMI is an important strategy for prognosis and is a key performance indicator. ${ }^{11}$

In 1 January 2017, the Integrated Heart Center team and emergency team of Cipto Mangunkusumo National General Hospital established a the CODE-STEMI. The aim of this study was to identify the effect of applying the CODE-STEMI protocol on in-hospital clinical outcomes such as MACE and mortality and the cost efficiency generated by this action.

\section{Methods}


This was a quantitative, interventional, retrospective cohort study. This study was conducted at Cipto Mangunkusumo National General Hospital in Jakarta as a National Refferal Centre in Indonesia. The medical records of all patients with a diagnosis of STEMI and who underwent PPCl from January 1st, 2015 to December 31st, 2018 were reviewed. Patients with MACE and severe comorbidity at initial presentation (acute stroke, hepatic cirrhosis, chronic inflammation disease sepsis, autoimmune, malignancy) were excluded from the study. Patients with incomplete medical record information were also excluded. Using the sample size formula for cohort study, we found the minimum amount of the sample to be 128 subjects. The total number of eligible patients in this study was 207 , which was further classified into two groups. The first group was the pre-CODE STEMI group, which consisted of patients presented from 2015-2016, and the second group was the CODE STEMI group, consisting of eligible patients from 2017-2018.

\section{Data collection and processing}

Data was obtained from the hospital's medical record database from their admission in emergency department, $\mathrm{PPCl}$ in catheterization laboratory, and post procedure care in ward. All patient who came to ED will go through triage first. Patients will then be examined by ED physician, and if typical chest pain and ST elevation on electrocardiogram reading is found, CODE STEMI will be activated. ED physician will call the on call cardiologist to confirm STEMI diagnosis, then will alert the interventional cardiologist, catheterization laboratory team, and administrative staff simultaneously. Patient will then be transferred immediately to catheterization laboratory to be performed PPCl. Each steps are regulated meticulously and each team has a clearly defined role in the protocol. The application of CODE STEMI is supervised closely and evaluated every three months. The CODE STEMI protocol is shown in Fig. 1. This study was approved by the Ethics Committee Faculty of Medicine University of Indonesia and ethical approval no. KET-634/UN2.F10/PPM.00.02/2019 was issued on 11th October 2019.

The primary end points were MACE rate (including stroke, cardiogenic shock, congestive heart failure, lethal dysrrhythmia and pericarditis) and mortality rate. The secondary end points were Length of Stay (LOS) and total hospitalization cost for measuring cost efficiency analysis. Total cost is converted from Indonesian Rupiah (IDR) to US Dollar (USD) currency. We also measured median D2BT from the patient's arrival at the emergency department to first balloon inflation and the duration of hospitalization. In 2017, European Heart Society guideline replaced the term 'door-to-balloon' time to 'diagnosis to wire crossing'

time as a clinical performance measure. ${ }^{5}$ However, in this study, we still adhere to the old terminology to maintain data consistency from pre CODE STEMI era.

\section{Data Analysis}

The data was coded and entered into an excel data sheet and was analyzed using SPSS, version 20.0 (IBM, Armonk, NY, United States of America). A univariate analysis was conducted initially and for selected variables, and bivariate analysis was conducted subsequently. We used chi square $\left(x^{2}\right)$ test to compare the MACE and mortality rate between each group. To identify the correlation between CODE CTEM Ml with dnorta hallonn timn I nc and anct wen wand Mann Whitney test. A 95\% confidence interval Loading [MathJax]/jax/output/CommonHTML/fonts/TeX/fontdata.js 
was used for all normally distributed data, while median and interquartile ranges were used for nonnormally distributed data.

\section{Result}

\section{Patient characteristics}

A total of 358 patients presented with STEMI and underwent PPCI between January 2015 and December 2018. There was 207 patients meet the inclusion criteria and were studied retrospectively. The cohort was divided into two groups. The first group consisted of 72 patients who were treated before the establishment of the CODE STEMI (mean age, $59.7 ; 87 \%$ were men). The second group consisted of 135 patients treated according to the CODE STEMI program that was initiated in January 2017 (mean age, $56.1 ; 86 \%$ were men). Risk factors for cardiovascular disease such as diabetes mellitus, hypertension, dyslipidemia, obesity, acute kidney failure, chronic kidney failure are distributed evenly in both the CODE STEMI group and the Pre CODE STEMI group. The baseline and clinical characteristics in both groups were similar, with no discernible discrepancies. (Table 1). 
Table 1

Baseline characteristics of the patients

\begin{tabular}{|c|c|c|c|}
\hline Characteristic & CODE STEMI $(\mathrm{N}=135)$ & Pre CODE STEMI ( $\mathrm{N}=72)$ & P Value \\
\hline Sex, n (\%) & & & 0,75 \\
\hline Male & $116(85,9)$ & $63(87,5)$ & \\
\hline Female & $19(14,1)$ & $9(12,5)$ & \\
\hline Age & 56,1 & 59,7 & 0,031 \\
\hline Risk Factor & $55(40,7)$ & $34(47,2)$ & 0,3696 \\
\hline \multicolumn{4}{|l|}{ Diabetes Mellitus, n (\%) } \\
\hline Hypertension, n (\%) & $74(54,81)$ & $41(56,94)$ & 0,7690 \\
\hline Dyslipidemia, n (\%) & $51(37,8)$ & $24(33,3)$ & 0,526 \\
\hline Obesity, n (\%) & $9(6,7)$ & $2(2,8)$ & 0,2348 \\
\hline Acute Kidney Injury, n (\%) & $23(17,0)$ & $16(22,5)$ & 0,3384 \\
\hline Chronic Kidney Disease, n (\%) & $18(13,43)$ & $22(30,56)$ & 0,0031 \\
\hline GRACE Score, n (\%) & & & 0,944 \\
\hline Severe & $4(21,05)$ & $3(18,92)$ & \\
\hline Moderate & $3(15,79)$ & $3(16,22)$ & \\
\hline Mild & $12(63,16)$ & $12(66,67)$ & \\
\hline Onset, n (\%) & & & 0,631 \\
\hline$>6$ hours & $22(16)$ & $55(76,3)$ & \\
\hline$\leq 6$ hours & $23(17)$ & $68(94)$ & \\
\hline
\end{tabular}

\section{Door to Baloon Time}

In the Pre CODE STEMI group, the median D2BT was 288 min (interquartile range [IQR] 120-1376). On the other hand, Table 3 shows CODE STEMI group had a significantly lower D2BT with a median of $158 \mathrm{~min}($ IQR $66-640)$, representing a $130 \mathrm{~min}(45 \%)$ improvement $(\mathrm{p}<0.01)$. The median D2BT has significantly starting from 2017. In 2015 and 2016, the median D2BT was still longer than 250 min while starting from 2017, the median D2BT doesn't exceed 160 min mark.

\section{In-hospital Clinical Outcomes}

Data analysis result showed no significant different between the two group in MACE and mortality rate. However, as seen in Table 2, we noticed a reduction of in-hospital MACE and mortality rate in CODE

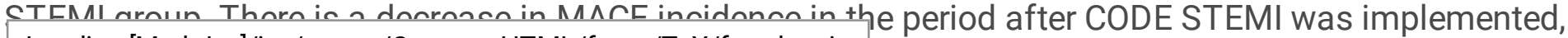
Loading [MathJax]/jax/output/CommonHTML/fonts/TeX/fontdata.js 
with risk ratio of 0.77 . The MACE rate was reduced by $10.83 \%$ from $48.61 \%$ in pre CODE STEMI period to $38.78 \%$ after the implementation of CODE STEMI program. The most common MACE that can be found in both groups are heart failure. We can also see reduction in mortality from $8.33 \%$ in pre CODE STEMI group to $4.44 \%$ in CODE STEMI group. The decrease in the percentage of these two parameters was not statistically significant, but clinically showed a decrease in the incidence of MACE and mortality in the group of patients who were treated using the STEMI CODE program ( $R R=0.777$ and 0.533 respectively).

Table 2

In hospital MACE and mortality in CODE STEMI and pre CODE STEMI group

\begin{tabular}{|c|c|c|c|}
\hline & $\begin{array}{l}\text { CODE STEMI } \\
\mathrm{n}=135\end{array}$ & $\begin{array}{l}\text { Pre CODE STEMI } \\
\mathrm{n}=72\end{array}$ & $\mathrm{RR}(\mathrm{Cl} 95 \%)$ \\
\hline MACE & $51(37,78 \%)$ & $35(48,61 \%)$ & $0,777(0,564-1,071)$ \\
\hline Mortality & $6(4,44 \%)$ & $6(8,33 \%)$ & $0,533(0,178-1,593)$ \\
\hline \multicolumn{4}{|c|}{ MACE = Major Adverse Cardiac Events; $\mathrm{RR}=$ Risk Ratio $\mathbf{C l}=$ Confidence Interva } \\
\hline
\end{tabular}

The median length of stay in the CODE STEMI group was 6 days with a standard deviation of 3.8 days, whereas for the Pre CODE STEMI group 7 days with a standard deviation of 3.5 days. The difference in length of stay for 1 day was statistically significant $(p=0.009)$. Figure 3 shows patient length of stay per year which tends to decrease after the CODE STEMI program is applied. Starting from 2017, the median length of stay of STEMI patients are maintained in 6 days, while the 2015 and 2016 median length of stay is 7.5 and 6.5 days respectively.

\section{Total hospital cost}

The average hospital bill in the CODE STEMI group was USD 3,818.95 while for the Pre CODE STEMI group was USD 4870.97. A significant difference of USD 1,027.16 (21\%) was found between the total costs of the two groups. There is a declining trend of total hospital cost each year from 2015 to 2018 especially after the implementation of CODE STEMI, from 5066 USD in 2015, 4746 USD in 2016, 4278 USD in 2017, to 3589 USD in 2018. 
Table 3

Data Analysis : Door to Balloon Time, Total Cost, Length of Stay

\begin{tabular}{|llll|}
\hline Variable & $\begin{array}{l}\text { CODE STEMI } \\
\mathbf{n = 1 3 5}\end{array}$ & $\begin{array}{l}\text { Pre CODE STEMI } \\
\mathbf{n = 7 2}\end{array}$ & $\mathbf{P}$ \\
\hline $\begin{array}{l}\text { D2BT, minutes } \\
\text { (min-max) }\end{array}$ & $158(66-640)$ & $288(120-1376)$ & $<0,001$ \\
\hline $\begin{array}{l}\text { Total cost, USD } \\
\text { (min-max) }\end{array}$ & $3,818.95$ & $4,870.97$ & $<0,001$ \\
\hline $\begin{array}{l}\text { Length of stay, days } \\
\text { (min-max) }\end{array}$ & $6(2-34)$ & $(1,690.55-16828,63)$ & \\
\hline D2BT, Door to balloon time; USD, US Dollar & & 0,009 \\
\hline
\end{tabular}

\section{Discussion}

We found that the introduction of CODE STEMI at our institution has successfully improved D2BT and reduced in-hospital MACE and mortality. In our study, the implementation of CODE STEMI resulted in $45 \%$ (130 min) improvement of D2BT, from $288 \mathrm{~min}$ to $158 \mathrm{~min}$. This result is similar to a study in Australia and Saudi Arabia which showed a 22.1 min and 30.5 min reduction of D2BT in post CODE STEMI period. ${ }^{11,12}$ Previous studies have revealed that prolonged D2BT for patients with STEMI was associated with increased mortality. ${ }^{13-15}$ The target of D2BT in STEMI patients undergoing PPCl according to American heart Association and European Society of Cardiology (ESC) is $\leq 90 \mathrm{~min} .{ }^{16}$ Although we have not reached the international standards, the CODE STEMI program has significantly reduced the door to balloon time, since even small reduction in D2BT has become of critically important in reducing mortality risk. ${ }^{17}$ The D2BT of less than 90 minutes has been shown to be associated with a reduction in major inhospital complications. ${ }^{18,19}$

We were able to observe a decrease in the incidence of MACE in STEMI patients by $10.83 \%$ in CODE STEMI group with risk ratio 0.78 . This means that there is a 0.78 risk of MACE in STEMI patients who are not treated using CODE STEMI protocol. Although the decrease was not proven to be statistically significant, but these results still show a downward trend in the incidence of MACE in STEMI patients, which is still an important value in clinical practice. This results are consistent with one study that reported a non statistically significant decrease in the incidence of MACE 30 days (5\% reduction) and 12 months MACE (10.1\% reduction) by the activation of CODE STEMI program. ${ }^{11}$ Another study in 2018 also reported lower complications in patients treated with the STEMI CODE program, specifically the incidence of recurrent infarction which drops to $8 \%$. $(p=0.043) .^{12}$ 
In alignment with MACE, there was a decrease in mortality by around $4 \%$ after the implementation of the CODE STEMI program with risk ratio 0.53 . Clinically, this means there is a reduced risk of mortality in STEMI patients treated using by the CODE STEMI. A study conducted in Canada, also showed a decrease in hospital mortality from $10-4.7 \%$ in the first year the CODE STEMI was applied. ${ }^{20}$ Every minute of delay in primary angioplasty for STEMI affects 1-year mortality, even after adjustment for baseline characteristics. ${ }^{21}$ Both result means that CODE STEMI protocol can reduce risk of mortality and MACE in STEMI patients. This is closely related to the reduction of D2BT after CODE STEMI was implemented. Shorter D2BT, which prompt early revascularization, has been associated with improved clinical outcomes in STEMI patients. ${ }^{17,22,23}$

The one day drop in length of stay of STEMI patients after the implementation of CODE STEMI program is consistent with a study in a teaching hospital in New Jersey, which found that during the first year CODE STEMI implementation, the average length of stay of patients dropped from 4 days to 3 days. ${ }^{24}$ Other study from Le May also stated that 1 year implementation of CODE STEMI as a city-wide program in Ottawa successfully reduced hospital length of stay to only 4 days.

The reduction of MACE, mortality, and length of stay contributes to the reduction of total hospitalization cost of STEMI patients. Lower treatment cost is required when patients don't have to go through extensive treatment and stay too long in the hospital after PPCl. Our study found that the implementation of CODE STEMI program has successfully reduced total hospital cost up to $21 \%$.

Efficiency is the ability to reduce service costs without reducing the benefits of services provided to patients, whereas optimalization is when an effective service is accomplished with the lowest expenditure of resources. (Donabedian, 2003) The results of data analysis concluded that the total hospital cost was reduced after the implementation of the CODE STEMI program. The benefits of CODE STEMI provided to patients can be seen from the improvement of the length of stay, door to balloon time, MACE, and mortality, which makes CODE STEMI an effective program as it yield a better clinical outcome.

$$
\text { Efficiency }=\frac{\text { ServiceBenefit }}{\text { ServiceCost }}
$$

According to the above efficiency formula, the improvement of clinical outcome and reduction of total hospitalization cost showed that this program has successfully improved the cost efficiency of STEMI treatment. Furthermore, CODE STEMI may also be considered as an optimal intervention for STEMI patients for its ability to provide an effective treatment with the lowest cost.

Providing timely emergency $\mathrm{PCl}$ is a complex undertaking demanding rapid coordination of care by multiple physicians, nurses and hospital staff. In 2006, the D2B alliance was launched, listing emergency physician-initiated Code STEMI as a method to reduce the door to balloon time. ${ }^{25}$ The success of CODE STEMI program in reducing D2BT thus reducing the in-hospital MACE and mortality in our institution was made possible by changing the system that previously required a step-by-step serial processes to a 
call, the interventional cardiologist on call, and the administrative and clerical staff. This strategy has been shown to reduce transfer time from ED to catheterization laboratory and the arrival of catheterization laboratory team.

In our study, the mean age of presentation was 57 years, which is almost a decade earlier than that reported in several other studies. ${ }^{26,27}$ It highlights the fact that people tend to get STEMI at younger age these days, especially in our country. Moreover, our patients have a high prevalence of risk factors for coronary artery diseases especially hypertension, diabetes mellitus, and dyslipidemia. This emphasizes the increased incidence of metabolic syndrome as a risk factor of atherosclerosis, which is the main cause of most STEMI. Therefore, a well-designed program to deal with the consequences is very much needed. Our country established a universal health coverage insurance program from the government so the cost efficiency of the program is also an important aspect to be aware of to maintain the national healthcare financial stability.

Although the number of patients included in this study is relatively small, the trends in favourable outcomes are encouraging and generalisable to most healthcare organisations, especially for general hospitals in developing country. The results of this study reveal that the CODE STEMI program will provide immense benefit when implemented in general hospitals where there are myriad patients with multivarious diseases, due to its optimal result albeit easy implementation and low cost. A larger sample size might be able to reveal a significant differences in the in-hospital MACE and mortality between the two groups.

\section{Limitation}

Our study had several limitations. First of all, it is a retrospective cohort study without randomization to either CODE STEMI or Pre CODE STEMI group. Our study is a also single-center study with data collected specific to our institution, so the result might not reflect the performance of other general hospitals. Furthermore, there might be confounding factors that could affect the clinical outcomes between the year 2015 to 2018, such as physician and interventional cardiologist clinical experience and skills which can improve throughout the years.

\section{Conclusion}

The implementation of CODE STEMI program in general hospital was associated with a significant improvement of in-hospital clinical outcome in the form of reduction of MACE and mortality rate in STEMI patients. The program is also able to reduce the length of stay of STEMI patients and is proven to improve cost efficiency of STEMI management. This re-emphasize the significance of a systematic program with early catheterization laboratory activation and concomitant early reperfusion in patients with STEMI, especially in general hospital. 
ACS

Acute Coronary Syndrome

$\mathrm{CHD}$

Coronary Heart Disease

D2BT

Door to Balloon Time

ED

Emergency Department

IDR

Indonesian Rupiah

IQR

Interquartil Range

LOS

Length of Stay

MACE

Major Adverse Cardiac Event

NCDs

Non-Communicable Disease

$\mathrm{PPCl}$

Primary Percutaneous Coronary Intervention

STEMI

ST Elevation Myocardial Infarction

UAP

Unstable Angine Pectoris

USD

United States Dollar

\section{Declarations}

\section{Ethics approval and consent to participate}

This study was approved by the Ethics Committee Faculty of Medicine University of Indonesia and ethical approval no. KET-634/UN2.F10/PPM.00.02/2019

\section{Consent for Publication}

Not Applicable

\section{Availability of Data and Materials}


The raw datasets used and/or analysed during the current study are available from the corresponding author on reasonable request

\section{Competing Interests}

The authors declare that they have no competing interests.

\section{Funding}

No funding was received for the research.

\section{Author's Contribution}

All authors contributed to the study design. EG conceived the study, performed the statistical analysis, and was the principal author of the paper. AS, IA, WS' ES, AW, LL: helped to interpret the data and draft the manuscript. All authors read and approved the final manuscript

\section{Acknowledgement}

None

\section{References}

1. Organization WH. Global Health Estimates 2016: Deaths by Cause, Age, Sex, by Country and by Region, 2000-2016. Geneva: WHO, 2018. 2019.

2. Gaziano TA, Bitton A, Anand S, Abrahams-Gessel S, Murphy A. Growing epidemic of coronary heart disease in low- and middle-income countries. Curr Probl Cardiol [Internet]. 2010 Feb;35(2):72-115. Available from: https://pubmed.ncbi.nlm.nih.gov/20109979.

3. Mensah GA, Roth GA, Fuster V. The Global Burden of Cardiovascular Diseases and Risk Factors. J Am Coll Cardiol [Internet]. 2019 Nov 19;74(20):2529 LP - 2532. Available from: http://www.onlinejacc.org/content/74/20/2529.abstract.

4. Mandelzweig L, Battler A, Boyko V, Bueno H, Danchin N, Filippatos G, et al. The second euro heart survey on acute coronary syndromes: Characteristics, treatment, and outcome of patients with ACS in Europe and the Mediterranean Basin in 2004. Eur Heart J. 2006;27(19):2285-93.

5. Ibanez B, James S, Agewall S, Antunes MJ, Bucciarelli-Ducci C, Bueno H, et al. 2017 ESC Guidelines for the management of acute myocardial infarction in patients presenting with ST-segment elevation: The Task Force for the management of acute myocardial infarction in patients presenting with STsegment elevation of the European Socie. Eur Heart J. 2017;39(2):119-77. 
Lars N-C, Jacob L, HD E, AK A, BL E, Steffen H, et al. Benefit From Reperfusion With Primary Percutaneous Coronary Intervention Beyond 12 Hours of Symptom Duration in Patients With STSegment-Elevation Myocardial Infarction. Circ Cardiovasc Interv [Internet]. 2018 Sep 1;11(9):e006842. Available from: https://doi.org/10.1161/CIRCINTERVENTIONS.118.006842.

7. Bloom DE, Cafiero ET, Jane-Llopis E, Abrahams-Gessel S, Bloom LR, Fathima S, et al. The Global Economic Burden of Non-communicable Disease. Geneva; 2011.

8. Jan S, Lee SW-L, Sawhney JPS, Ong TK, Chin CT, Kim H-S, et al. Predictors of high-cost hospitalization in the treatment of acute coronary syndrome in Asia: findings from EPICOR Asia. BMC Cardiovasc Disord [Internet]. 2018 Jul 4;18(1):139. Available from: https://pubmed.ncbi.nlm.nih.gov/29973147.

9. Page RL, II VG, Van Den Bos J, Gray TJ, Hoetzer GL, Bhandary D, et al. The cost of inpatient death associated with acute coronary syndrome. Vasc Health Risk Manag. 2016;12:13.

10. $10.1155 / 2019 / 7562637$

Habib H, Ginanjar E, Mansjoer A, Sulistio S, Albar IA, Mulyana RM. ST-Elevation Myocardial Infarction: A Simulation Case for Evaluation of Interprofessional Performance in a Hospital. Morita S, editor. Emerg Med Int [Internet]. 2019;2019:7562637. Available from:

https://doi.org/10.1155/2019/7562637.

11. Koh JQ, Tong DC, Sriamareswaran R, Yeap A, Yip B, Wu S, et al. In-hospital “CODE STEMI” improves door-to-balloon time in patients undergoing primary percutaneous coronary intervention.'. Emerg Med Australas. 2018 Apr;30(2):222-7.

12. Alyahya AA, Alghammass MA, Aldhahri FS, Alsebti AA, Alfulaij AY, Alrashed SH, et al. The impact of introduction of Code-STEMI program on the reduction of door-to-balloon time in acute ST-elevation myocardial infarction patients undergoing primary percutaneous coronary intervention: A singlecenter study in Saudi Arabia. J Saudi Hear Assoc. 2018 Jul;30(3):172-9.

13. Rathore SS, Curtis JP, Chen J, Wang Y, Nallamothu BK, Epstein AJ, et al. Association of door-toballoon time and mortality in patients admitted to hospital with ST elevation myocardial infarction: national cohort study. Bmj. 2009;338:b1807.

14. McNamara RL, Herrin J, Bradley EH, Portnay EL, Curtis JP, Wang Y, et al. Hospital improvement in time to reperfusion in patients with acute myocardial infarction, 1999 to 2002. J Am Coll Cardiol. 2006;47(1):45-51.

15. McNamara RL, Herrin J, Wang Y, Curtis JP, Bradley EH, Magid DJ, et al. Impact of delay in door-toneedle time on mortality in patients with ST-segment elevation myocardial infarction. Am $\mathrm{J}$ Cardiol. 2007;100(8):1227-32.

16. 10.1161/CIR.0b013e3182742c84

O'Gara PT, Frederick GK, Deborah DA, Donald EC, K CM, A. de LJ, et al. 2013 ACCF/AHA Guideline for the Management of ST-Elevation Myocardial Infarction: Executive Summary. Circulation [Internet]. 2013 Jan 29;127(4):529-55. Available from: https://doi.org/10.1161/CIR.0b013e3182742c84. 
17. McNamara RL, Wang Y, Herrin J, Curtis JP, Bradley EH, Magid DJ, et al. Effect of door-to-balloon time on mortality in patients with ST-segment elevation myocardial infarction. J Am Coll Cardiol. 2006;47(11):2180-6.

18. AlHabib KF, Sulaiman K, Al Suwaidi J, Almahmeed W, Alsheikh-Ali AA, Amin H, et al. Patient and system-related delays of emergency medical services use in acute ST-elevation myocardial infarction: results from the third gulf registry of acute coronary events (Gulf RACE-3Ps). PLoS One. 2016;11(1).

19. Shiomi H, Nakagawa Y, Morimoto T, Furukawa Y, Nakano A, Shirai S, et al. Association of onset to balloon and door to balloon time with long term clinical outcome in patients with ST elevation acute myocardial infarction having primary percutaneous coronary intervention: observational study. Bmj. 2012;344:e3257.

20. Le May M. Code STEMI. implementation of a city-wide program for rapid assessment and management of myocardial infarction. Can Med Assoc J. 2009 Oct;181(8):E136-7.

21. De Luca G, Suryapranata H, Ottervanger JP, Antman EM. Time Delay to Treatment and Mortality in Primary Angioplasty for Acute Myocardial Infarction: Every Minute of Delay Counts. Circulation. 2004;109(10):1223-5.

22. Cannon CP, Gibson CM, Lambrew CT, Shoultz DA, Levy D, French WJ, et al. Relationship of symptomonset-to-balloon time and door-to-balloon time with mortality in patients undergoing angioplasty for acute myocardial infarction. Jama. 2000;283(22):2941-7.

23. Berger PB, Ellis SG, Holmes DR Jr, Granger CB, Criger DA, Betriu A, et al. Relationship between delay in performing direct coronary angioplasty and early clinical outcome in patients with acute myocardial infarction: results from the global use of strategies to open occluded arteries in Acute Coronary Syndromes (GUSTO-IIb) trial. Circulation. 1999;100(1):14-20.

24. Parikh R, Faillace R, Hamdan A, Adinaro D, Pruden J, DeBari V, et al. An emergency physician activated protocol, 'Code STEMI" reduces door-to-balloon time and length of stay of patients presenting with ST-segment elevation myocardial infarction.'. Int J Clin Pract. 2009 Mar;63(3):398406.

25. Nissen SE, Brush JE, Krumholz HM. President's page: GAP-D2B: an alliance for quality. Journal of the American College of Cardiology; 2006.

26. 10.1161/JAHA.119.013296

Vernon ST, Sean C, Mario D, CC K, Jens K, Karice H, et al. ST-Segment-Elevation Myocardial Infarction (STEMI) Patients Without Standard Modifiable Cardiovascular Risk Factors-How Common Are They, and What Are Their Outcomes? J Am Heart Assoc [Internet]. 2019 Nov 5;8(21):e013296. Available from: https://doi.org/10.1161/JAHA.119.013296.

27. Terkelsen CJ, Lassen JF, Nørgaard BL, Gerdes JC, Jensen T, Gøtzsche LB-H, et al. Mortality rates in patients with ST-elevation vs. non-ST-elevation acute myocardial infarction: observations from an unselected cohort. Eur Heart J. 2004;26(1):18-26. 
Figures

\section{Patient Arrival}

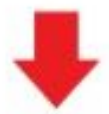

ED Triage

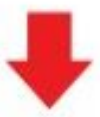

Assessment by ED physician

Typical Chest pain, ST elevation on ECG

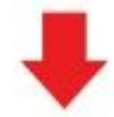

\section{Contact cardiologist consultant}

Conf rm STEMI diagnosis to activate CODE STEMI

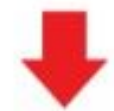

\section{ALERT}

\section{Interventional Cardiologist}

2.Cardiac cathetherization laboratory team

3. Clerical and administrative staf

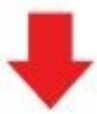

\section{Patient transfer to \\ Primary $\mathrm{PCl}$} catheterization laboratory

Figure 1

CODE STEMI protocol in Cipto Mangunkusumo Hospital. CODE STEMI provide a structured step-by step process of STEMI management. Once the CODE STEMI is activated, physician alert the interventional 
the preparation of primary percutaneous intervention. Abbreviation : ECG,Electrocardiogram; ED, Emergency department; PCl,primary cutaneous intervention; STEMI, ST elevation myocardial infarction

\section{Supplementary Files}

This is a list of supplementary files associated with this preprint. Click to download.

- Totalcosttrend.pdf

- Doortoballoontrend.pdf

- Lengthofstaytrend.pdf 